\title{
Virtual Morphometry of the First Lumbar Vertebrae for Estimation of Sex Using Computed Tomography Data in the Turkish Population
}

\author{
Mustafa Bozdag $^{1}$, Gokce Karaman ${ }^{2}$ \\ 1. Radiology, Tepecik Training and Research Hospital, Izmir, TUR 2. Department of Forensic Science, Council of \\ Forensic Medicine, Manisa, TUR
}

Corresponding author: Mustafa Bozdag, bozdagmustafa.84@gmail.com

\begin{abstract}
Introduction

It may be necessary to make sex estimation by examining other bones that have been obtained intact. Vertebrae, especially the thoracic and lumbar vertebrae, are among the best-preserved skeletal elements from the forensic and archaeological point of view. Therefore, lumbar vertebrae can become an important skeletal element for sex estimation. In our study, measurements were made on the CT images of the first lumbar vertebra, and the accuracy of sex estimation from the L1 vertebra was investigated in the Turkish population.
\end{abstract}

\section{Materials and methods}

Three dimensional (3D) models of the L1 vertebra were created from CT images of 241 (121 females and 120 males) individuals. Twenty-two linear measurements were taken in lateral, anterior, and superior views of 3D models of the L1 vertebra. Univariate and multivariate discriminant function analyzes were applied to the measured parameters to determine predictive rates of sex. Intra- and interobserver errors were calculated.

\section{Results}

All linear measurements were higher in males than females. All parameters excluding SCD (Spinal canal depth), PLu (Upper pedicle length), PLI (Lower pedicle length), AHi (Inferior articular process height), and SPH (Spinous process height) showed statistically significant differences between sex. The highest rate of $70.5 \%$ was obtained for the EPWu (Upper end-plate width) and EPWl (Lower end-plate width) parameters. When all variables of $\mathrm{L} 1$ vertebra were included in the stepwise discriminant analysis, correct prediction rates were determined as $72.6 \%$.

\section{Conclusion}

Review began 06/25/2021 Review ended 07/16/2021 Published 07/23/2021

๑) Copyright 2021

Bozdag et al. This is an open access article distributed under the terms of the Creative Commons Attribution License CC-BY 4.0., which permits unrestricted use, distribution, and reproduction in any medium, provided the original author and source are credited.
Our study is the first study in which L1 vertebrae are examined with the purpose of sex estimation in the Turkish population and we think that our data will be an important reference for sex estimation from the L1 vertebra in the Turkish population.

\section{Categories: Radiology, Other}

Keywords: lumbar, morphometry, tomography, vertebrae, turkish

\section{Introduction}

Identification of skeletal, severely degraded, or unidentified human remains is important for forensic and humanitarian reasons [1-3]. In the identification process; age, sex, stature, and other unique features of the person are determined. Since other analyses are sex-dependent methods, sex estimation is usually the first step in the biological identification process [4-5].

Although the most reliable results in sex estimation with morphological and metric methods are obtained from the analyses of the hip and skull bones; in cases such as natural disasters and mass disasters, it may not always be possible to examine the skull and hip bones due to animal activities, voluntary damage and deterioration of the skeleton due to taphonomic processes [2-3]. It may be necessary to estimate sex by examining any intact bones that have been obtained. For this reason, it is necessary to develop different methods for sex estimation with bones other than hip and skull bones. Today, many parts of the adult skeleton are used for sex estimation [3]. Studies on the vertebral column have also shown that the vertebrae demonstrate sexual dimorphic features [6-15].

Lumbar vertebrae are the largest of the vertebrae and usually consist of five vertebrae. They have a larger 
vertebral body due to their load-bearing properties [16]. They have no costal facets and transverse foramina. The vertebral foramen is triangular in shape and is larger compared to the thoracic vertebrae, but smaller than the cervical vertebrae [16]. Vertebrae, especially the thoracic and lumbar vertebrae, are among the bestpreserved skeletal elements from the forensic and archaeological point of view [17-19]. Therefore, lumbar vertebrae can become an important skeletal element for sex estimation.

Zheng et al. stated that sex estimation can be made with an accuracy of $88.6 \%$ using discriminant function analysis from the L1 vertebra [11]. Ostrofsky and Churchill also stated that among the lumbar vertebrae, the L1 was the most sex diagnostic vertebra with an accuracy rate of $87.1 \%$ for sex estimation [12]. Decker et al. reported that each of the lumbar vertebrae demonstrated sexual dimorphism exceeding $80 \%$ using discriminant function analysis [14]. Azofra-Monge and Alemán Aguilera in their study evaluating the lumbar vertebrae stated that the lumbar vertebrae showed more sexual dimorphism in their study compared to previous studies, and the highest accuracy rates in the regression equations were obtained from the L1 vertebra (90.1-94.5\%) [15]. Studies show that sex estimation can be made using lumbar vertebrae with high accuracy rates [11-12,14-15]. It has also been shown in studies that, of the five lumbar vertebrae, especially the first lumbar vertebra is more sexually dimorphic than the other lumbar vertebrae $[12,14]$.

The degree of sexual dimorphism present in postcranial skeletal elements may differ between populations, therefore, the most accurate sex marker may differ between populations [20]. It is stated that vertebral discriminant function analyzes used in sex estimation are also population-specific [17]. For this reason, when sex estimation is made using vertebrae, employing data obtained from studies conducted on that particular population will increase the accuracy of sex estimation.

There is no study in the Turkish population that evaluated the lumbar vertebrae in terms of sex predicting accuracy. In our study; lumbar computed tomography (CT) images were examined, measurements were made on the first lumbar vertebra, and the accuracy of sex estimation from L1 vertebra in the Turkish population was investigated by applying discriminant function analysis.

\section{Materials And Methods}

This retrospective study was approved by the Hospital Research Ethics Committee (protocol number: 2020/11-29) and the study was conducted in accordance with the standards of the Declaration of Helsinki. This study was conducted on 241 people (121 females and 120 males) from the west of Turkey. These individuals were patients who underwent CT scans without contrast medium because of urinary stone diseases from January 2016 to December 2020. First lumbar vertebra CT images of individuals were retrospectively evaluated. Cases that had any bone pathologies, degenerative bone diseases, fractured or deformed L1 vertebra were excluded from the study.

CT scans were performed using a 128-slice multi-detector computed tomography (MDCT) scanner (SOMATOM Definition Edge, Siemens Healthcare, Erlangen, Germany). Scan parameters were $100 \mathrm{kV}, 186$ $\mathrm{mA}$, section thickness $1 \mathrm{~mm}$, and reconstruction interval $0.6 \mathrm{~mm}$. All scan data were transferred from the archive to a workstation (Aquarius workstation, TeraRecon, San Mateo, CA, USA) to create threedimensional (3D) models of the L1 vertebra by post-processing procedures. Twenty-two linear measurements were taken in lateral, anterior, and superior views of 3D models of the L1 vertebra. The only left side of the parameter was measured for analyses except for transverse distance (TD). The measurements were performed by one radiologist with eleven years of experience in musculoskeletal radiology, one forensic pathologist with six years of experience in forensic anthropology. The measured parameters are shown in Figure 1 and listed in Table 1. 


\section{Cureus}

(a)

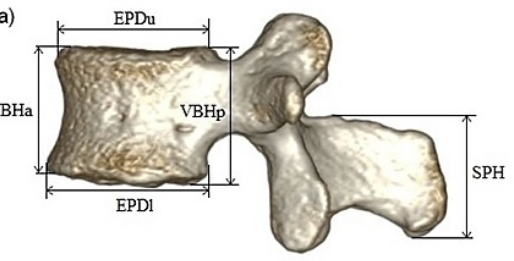

(c)

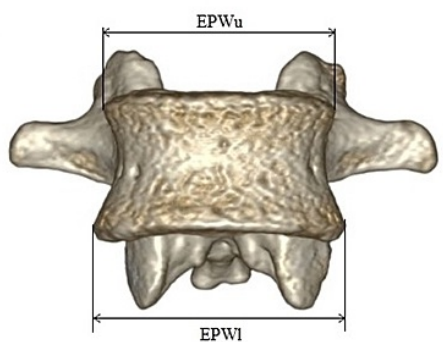

(e)

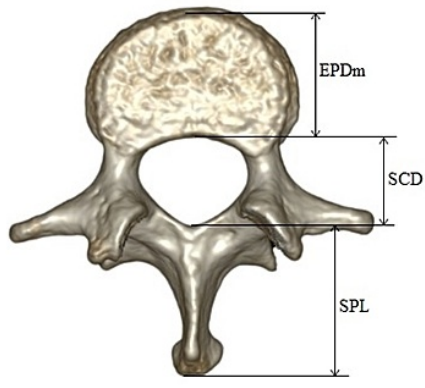

(b)

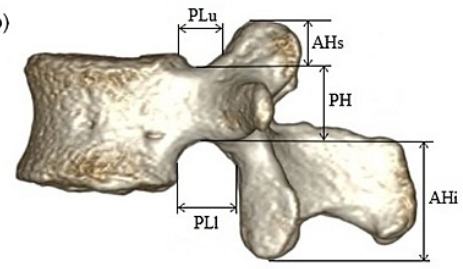

(d)

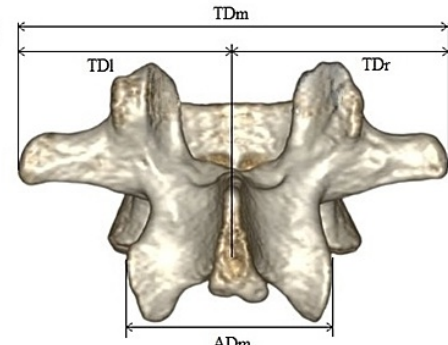

(f)

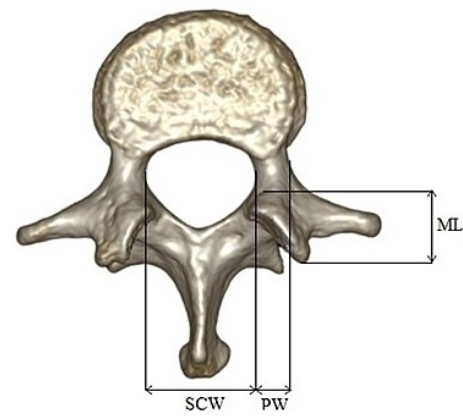

FIGURE 1: Three-dimensional views of L1 vertebra. (a) and (b) are lateral views; (c) and (d) are anterior and posterior views; (e) and (f) superior views.

Adapted from Zheng et al. [11]. Permission for the re-print was obtained. 


\section{Cureus}

\begin{tabular}{|c|c|c|}
\hline Vertebral part & Symbol & Definition \\
\hline \multirow{7}{*}{ Vertebral body } & EPDm & Middle end-plate depth (Figure 1e) \\
\hline & EPDu & Upper end-plate depth (Figure 1a) \\
\hline & EPDI & Lower end-plate depth (Figure 1a) \\
\hline & VBHa & Anterior height of vertebral body (Figure 1a) \\
\hline & VBHp & Posterior height of vertebral body (Figure 1a) \\
\hline & EPWu & Upper end-plate width (Figure 1c) \\
\hline & EPWI & Lower end-plate width (Figure 1c) \\
\hline \multirow{6}{*}{ Vertebral foramen pedicle } & SCW & Spinal canal width (Figure 1f) \\
\hline & SCD & Spinal canal depth (Figure 1e) \\
\hline & $\mathrm{PH}$ & Pedicle height (Figure 1b) \\
\hline & PW & Pedicle width (Figure 1f) \\
\hline & PLu & Upper pedicle length (Figure 1b) \\
\hline & PLI & Lower pedicle length (Figure 1b) \\
\hline \multirow{3}{*}{ Articular process } & AHs & Superior articular process height (Figure 1b) \\
\hline & $\mathrm{AHi}$ & Inferior articular process height (Figure 1b) \\
\hline & ADm & Maximum distance between articular processes (Figure 1d) \\
\hline Mammillary process & ML & Mammillary process length (Figure 1f) \\
\hline \multirow{2}{*}{ Transverse process } & TD $(r, l)$ & Transverse process distance (Figure 1d) \\
\hline & TDm & Maximum distance between transverse processes (Figure 1d) \\
\hline \multirow{2}{*}{ Spinous process } & SPH & Spinous process height (Figure 1a) \\
\hline & SPL & Spinous process length (Figure 1e) \\
\hline
\end{tabular}

\section{TABLE 1: Nomenclature and definition of the measurements.}

Derived from Zheng et al. [11].

The SPSS software (ver. 22; SPSS Inc., Chicago, IL) was used to assess the variables. After determining the mean and standard deviations for each variable, the differences between the genders were analyzed using Student's t-test. P values less than 0.01 were considered statistically significant. Univariate and multivariate discriminant function analyses were applied to the measured parameters to determine predictive rates of sex. After all, measurements were completed by a radiologist, all parameters of 20 randomly selected people were measured again by the first observer and by a second observer. Intra- and interobserver errors were calculated using technical error of measurement (TEM), relative TEM (rTEM), and the coefficient of reliability (R) of the measurement, as suggested by Ulijaszek and Kerr [21].

\section{Results}

In total, 241 subjects (121 females and 120 males) were enrolled in the study. The mean age of the total was $43 \pm 16.4$, the mean age of females was $44 \pm 16.6$, and the mean age of males was $41 \pm 15.9$. The mean and standard deviation values according to sex and P-values of the variables are shown in Table 2. All linear measurements were higher in males than females (Table 2). All parameters excluding SCD (Spinal canal depth), PLu (Upper pedicle length), PLI (Lower pedicle length), AHi (Inferior articular process height), and SPH (Spinous process height) showed statistically significant differences between sex $(\mathrm{p}<0.01)$.

\begin{tabular}{|l}
\hline Parameters \\
Middle end-plate depth (EPDm)
\end{tabular}

\begin{tabular}{|l|l|l|l|l|}
\hline Sex & N & Mean & SD & P \\
\hline Female & 120 & 3.055 & 0.324 & \\
\hline
\end{tabular}




\section{Cureus}

Upper end-plate depth (EPDu)

Lower end-plate depth (EPDI)

Anterior height of vertebral body (VBHa)

Posterior height of vertebral body (VBHp)

Upper end-plate width (EPWu)

Lower end-plate width (EPWI)

Spinal canal width (SCW)

Spinal canal depth (SCD)

Pedicle height $(\mathrm{PH})$

Pedicle width (PW)

Upper pedicle length (PLu)

Lower pedicle length (PLi)

Superior articular process height (AHs)

Inferior articular process height $(\mathrm{AHi})$

Maximum distance between articular processes (Adm)

Mammillary process length (ML)

Right transverse process distance (TDr)

Left transverse process distance (TDI)

Maximum distance between transverse processes (TDm)

\begin{tabular}{|c|c|c|c|c|}
\hline Male & 121 & 3.333 & 0.334 & \multirow{3}{*}{0} \\
\hline Female & 120 & 3.143 & 0.338 & \\
\hline Male & 121 & 3.492 & 0.365 & \\
\hline Female & 120 & 3.223 & 0.347 & \multirow{2}{*}{0} \\
\hline Male & 121 & 3.557 & 0.399 & \\
\hline Female & 120 & 2.921 & 0.25 & \multirow{2}{*}{0.002} \\
\hline Male & 121 & 3.028 & 0.289 & \\
\hline Female & 120 & 3.052 & 0.231 & \multirow{2}{*}{0} \\
\hline Male & 121 & 3.17 & 0.223 & \\
\hline Female & 120 & 4.049 & 0.376 & \multirow{2}{*}{0} \\
\hline Male & 121 & 4.45 & 0.422 & \\
\hline Female & 120 & 4.384 & 0.447 & \multirow{2}{*}{0} \\
\hline Male & 121 & 4793 & 0.447 & \\
\hline Female & 120 & 2.056 & 0.27 & \multirow{3}{*}{0} \\
\hline & & & & \\
\hline IVIale & 121 & 2.191 & 0.21 & \\
\hline Female & 120 & 1.653 & 0.257 & \multirow{2}{*}{0.64} \\
\hline Male & 121 & 1.67 & 0.287 & \\
\hline Female & 120 & 1.913 & 0.351 & \multirow{2}{*}{0.001} \\
\hline Male & 121 & 2.07 & 0.353 & \\
\hline Female & 120 & 0.89 & 0.226 & \multirow{2}{*}{0.001} \\
\hline Male & 121 & 0.991 & 0.236 & \\
\hline Female & 120 & 0.825 & 0.212 & \multirow{2}{*}{0.845} \\
\hline Male & 121 & 0.82 & 0.223 & \\
\hline Female & 120 & 1.207 & 0.265 & \multirow{2}{*}{0.771} \\
\hline Male & 121 & 1.218 & 0.28 & \\
\hline Female & 120 & 0.947 & 0.225 & \multirow{2}{*}{0.004} \\
\hline Male & 121 & 1.038 & 0.252 & \\
\hline Female & 120 & 2.866 & 0.679 & \multirow{2}{*}{0.059} \\
\hline Male & 121 & 3.039 & 0.732 & \\
\hline Female & 120 & 2.78 & 0.488 & \multirow{2}{*}{0.002} \\
\hline Male & 121 & 2.989 & 0.55 & \\
\hline Female & 120 & 2.275 & 0.421 & \multirow[b]{2}{*}{0} \\
\hline Male & 121 & 2.505 & 0.489 & \\
\hline Female & 120 & 3.652 & 0.421 & \multirow[b]{2}{*}{0} \\
\hline Male & 121 & 3.999 & 0.452 & \\
\hline Female & 120 & 3.494 & 0.425 & \multirow[b]{2}{*}{0} \\
\hline Male & 121 & 3.795 & 0.433 & \\
\hline Female & 120 & 7.008 & 0.656 & \multirow[b]{2}{*}{0} \\
\hline Male & 121 & 7.558 & 0.659 & \\
\hline Female & 120 & 2.641 & 0.276 & \\
\hline
\end{tabular}




\section{Cureus}

Spinous process height (SPH)

$\begin{array}{lllll}\text { Male } & 121 & 2.634 & 0.379 & 0.874 \\ \text { Female } & 120 & 2.848 & 0.33 & \\ \text { Male } & 121 & 3.119 & 0.428 & 0\end{array}$

Spinous process length (SPL)

TABLE 2: Means, standard deviations and P-values for mean differences between females and males for all L1 vertebra variables.

In calculating the correlations of the cases evaluated for intra-observer and inter-observer evaluation, for all the measured variables; correlations were found to be high in all measurement variables. $\mathrm{R}$ values ranged between $0.856-0.994$ for intra-observer evaluation and ranged between $0.855-0.997$ for the inter-observer evaluation. Technical measurement analysis results are presented in Table 3.

\begin{tabular}{|c|c|c|c|c|c|c|}
\hline \multirow{2}{*}{ Parameters } & \multicolumn{3}{|c|}{ Intra-observer (n:20) } & \multicolumn{3}{|c|}{ Inter-observer (n:20) } \\
\hline & TEM & rTEM & R & TEM & rTEM & R \\
\hline Middle end-plate depth (EPDm) & 0.114 & 3.409 & 0.939 & 0.114 & 3.460 & 0.939 \\
\hline Upper end-plate depth (EPDu) & 0.052 & 1.651 & 0.991 & 0.060 & 1.883 & 0.988 \\
\hline Lower end-plate depth (EPDI) & 0.050 & 1.452 & 0.986 & 0.081 & 2.354 & 0.962 \\
\hline Anterior height of vertebral body (VBHa) & 0.002 & 0.085 & 0.887 & 0.003 & 0.104 & 0.855 \\
\hline Posterior height of vertebral body (VBHp) & 0.002 & 0.081 & 0.977 & 0.004 & 0.161 & 0.910 \\
\hline Upper end-plate width (EPWu) & 0.039 & 0.904 & 0.993 & 0.092 & 2.152 & 0.962 \\
\hline Lower end-plate width (EPWI) & 0.107 & 2.294 & 0.951 & 0.116 & 2.490 & 0.941 \\
\hline Spinal canal width (SCW) & 0.094 & 4.436 & 0.856 & 0.060 & 2.844 & 0.936 \\
\hline Spinal canal depth (SCD) & 0.065 & 3.921 & 0.938 & 0.052 & 3.212 & 0.952 \\
\hline Pedicle height (PH) & 0.055 & 2.550 & 0.985 & 0.039 & 1.829 & 0.991 \\
\hline Pedicle width (PW) & 0.085 & 8.452 & 0.893 & 0.043 & 4.360 & 0.971 \\
\hline Upper pedicle length (PLu) & 0.056 & 7.115 & 0.949 & 0.037 & 4.766 & 0.977 \\
\hline Lower pedicle length (PLI) & 0.061 & 4.754 & 0.965 & 0.085 & 6.611 & 0.948 \\
\hline Superior articular process height (AHs) & 0.039 & 4.169 & 0.952 & 0.052 & 5.497 & 0.927 \\
\hline Inferior articular process height (AHi) & 0.063 & 2.439 & 0.994 & 0.047 & 1.840 & 0.997 \\
\hline Maximum distance between articular processes (ADm) & 0.067 & 2.234 & 0.977 & 0.115 & 3.905 & 0.925 \\
\hline Mammillary process length (ML) & 0.147 & 6.278 & 0.896 & 0.068 & 2.932 & 0.977 \\
\hline Right transverse process distance (TDr) & 0.173 & 4.552 & 0.910 & 0.060 & 1.558 & 0.989 \\
\hline Left transverse process distance (TDI) & 0.047 & 1.300 & 0.992 & 0.112 & 3.086 & 0.961 \\
\hline Maximum distance between transverse processes (TDm) & 0.157 & 2.170 & 0.953 & 0.093 & 1.281 & 0.983 \\
\hline Spinous process height (SPH) & 0.002 & 0.102 & 0.963 & 0.004 & 0.204 & 0.888 \\
\hline Spinous process length (SPL) & 0.129 & 4.342 & 0.867 & 0.071 & 2.386 & 0.952 \\
\hline
\end{tabular}

TABLE 3: Technical error measurement (TEM), relative TEM (rTEM) and $R$ for intra- and interobserver error for the variables included in the study.

Correct prediction rates of all single variables were calculated between $50.2 \%-70.5 \%$ to determine sex in the univariate discriminant analysis results of the variables measured for L1 vertebra. The highest rate of $70.5 \%$ 


\section{Cureus}

was obtained for the EPWu (Upper end-plate width) and EPWI (Lower end-plate width) parameters. Univariate discrimination analysis results are presented in Table 4.

\begin{tabular}{|c|c|c|c|c|}
\hline \multirow{2}{*}{ Variable } & \multirow{2}{*}{ Wilk's Lambda } & \multicolumn{3}{|c|}{ Correct Prediction Rates (\%) } \\
\hline & & Female & Male & Overall \\
\hline Middle end-plate depth (EPDm) & 0.847 & 72.5 & 65.3 & 68.9 \\
\hline Upper end-plate depth (EPDu) & 0.802 & 65 & 72.7 & 68.9 \\
\hline Lower end-plate depth (EPDI) & 0.833 & 70.8 & 65.3 & 68.5 \\
\hline Anterior height of vertebral body (VBHa) & 0.962 & 58.3 & 61.2 & 59.8 \\
\hline Posterior height of vertebral body (VBHp) & 0.936 & 62.5 & 49.6 & 56.0 \\
\hline Upper end-plate width (EPWu) & 0.798 & 74.2 & 65.3 & 70.5 \\
\hline Lower end-plate width (EPWI) & 0.825 & 74.2 & 66.9 & 70.5 \\
\hline Spinal canal width (SCW) & 0.936 & 65 & 54.5 & 59.8 \\
\hline Spinal canal depth (SCD) & 0.999 & 58.3 & 44.6 & 51.5 \\
\hline Pedicle height $(\mathrm{PH})$ & 0.952 & 62.5 & 61.2 & 61.8 \\
\hline Pedicle width (PW) & 0.954 & 62.5 & 54.5 & 58.5 \\
\hline Upper pedicle length (PLu) & 1 & 39.2 & 60.3 & 50.2 \\
\hline Lower pedicle length (PLI) & 1 & 55 & 45.5 & 50.2 \\
\hline Superior articular process height (AHs) & 0.966 & 61.7 & 47.9 & 54.8 \\
\hline Inferior articular process height (AHi) & 0.985 & 49.2 & 52.9 & 51.0 \\
\hline Maximum distance between articular processes (ADm) & 0.961 & 58.3 & 57.9 & 58.1 \\
\hline Mammillary process length (ML) & 0.940 & 64.2 & 58.7 & 62.2 \\
\hline Right transverse process distance (TDr) & 0.863 & 65.8 & 62.8 & 64.3 \\
\hline Left transverse process distance (TDI) & 0.890 & 64.2 & 65.3 & 64.7 \\
\hline Maximum distance between transverse processes (TDm) & 0.843 & 42 & 65.3 & 67.6 \\
\hline Spinous process height (SPH) & 1 & 48.3 & 57.9 & 53.1 \\
\hline Spinous process length (SPL) & 0.888 & 66.7 & 63.6 & 65.1 \\
\hline
\end{tabular}

TABLE 4: Accuracy rates of univariate discriminant analysis.

As a result of multivariate discriminant analysis for $\mathrm{L} 1$ vertebra, correct prediction rates were $73.9 \%$ for the vertebral body parameters, $66.8 \%$ for the vertebral foramen and pedicle parameters, and $69.7 \%$ for all vertebral process parameters. When all variables of L1 vertebra were included in the stepwise discriminant analysis, correct prediction rates were determined as $72.6 \%$. Multivariate discriminant analysis results were presented in Table 5 . 


\section{Cureus}

\begin{tabular}{|c|c|c|c|c|}
\hline \multirow{2}{*}{ Variable } & \multirow{2}{*}{ Wilk's Lambda } & \multicolumn{3}{|c|}{ Accuracy Rates (\%) } \\
\hline & & Female & Male & Overall \\
\hline Body $^{\star}$ & .693 & 71.9 & 75.8 & 73.9 \\
\hline \multicolumn{5}{|l|}{ Upper end-plate depth (EPDu) } \\
\hline \multicolumn{5}{|l|}{ Middle end-plate depth (EPDm) } \\
\hline \multicolumn{5}{|c|}{ Posterior height of vertebral body (VBHp) } \\
\hline \multicolumn{5}{|l|}{ Upper end-plate width (EPWu) } \\
\hline \multicolumn{5}{|l|}{ Lower end-plate width (EPWI) } \\
\hline Foramen and pedicle $e^{\star \star}$ & .847 & 63.6 & 70.0 & 66.8 \\
\hline \multicolumn{5}{|l|}{ Spinal canal width (SCW) } \\
\hline \multicolumn{5}{|l|}{ Pedicle height (PH) } \\
\hline \multicolumn{5}{|l|}{ Pedicle width (PW) } \\
\hline Process $^{\star \star \star}$ & .775 & 70.0 & 69.4 & 69.7 \\
\hline \multicolumn{5}{|l|}{ Spinous process length (SPH) } \\
\hline \multicolumn{5}{|l|}{ Mammillary process length (ML) } \\
\hline \multicolumn{5}{|c|}{ Maximum distance between transverse processes (TDm) } \\
\hline All Parameters & 0.985 & 75.8 & 69.4 & 72.6 \\
\hline \multicolumn{5}{|l|}{ Spinous process length (SPL) } \\
\hline \multicolumn{5}{|l|}{ Mammillary process length (ML) } \\
\hline \multicolumn{5}{|c|}{ Maximum distance between transverse processes (TDm) } \\
\hline \multicolumn{5}{|l|}{ Spinal canal width (SCW) } \\
\hline \multicolumn{5}{|l|}{ Upper end-plate width (EPWu) } \\
\hline Upper end-plate depth (EPDu) & & & & \\
\hline
\end{tabular}

TABLE 5: Accuracy rates of multivariate discriminant analysis.

\section{Discussion}

In our study, a significant sexual dimorphism was observed in 17 of 22 metric measurements made on the L1 vertebra. Not more than an $80 \%$ accuracy rate was observed for sex estimation in any of the metric measurements made on the L1 vertebra. The accuracy rates of these 17 metric measurements in which sexual dimorphism was detected varied between $50.2 \%$ and $70.5 \%$. EPWu and EPWI measurements had the highest sex predicting accuracy (70.5\%).

In the study of Ostrofsky and Churchill, L1 vertebra BSDVD (body superior dorsoventral diameter) and BSTD (body superior transverse diameter) measurements were found to have an accuracy rate of more than $80 \%$ [12]. In the study by Zheng et al. the EPWu measurement alone had the highest accuracy and EPWu, EPDm (middle end-plate depth), EPWI, EPDI (lower end-plate depth), EPDu (upper end-plate depth) measurements were found to have an accuracy rate exceeding $80 \%$ [11]. In the study of Decker et al. it is stated that only EPWu measurement has an accuracy rate of more than 80\% [14]. In our study, the metric measurements made on the vertebral body of the L1 vertebra were the most dimorphic measurements, and the results were compatible with previous studies in the literature. However, in our study, it was observed that the accuracy rates of measurements made on L1 vertebrae were lower accuracy compared to other studies.

In previous studies, it has been stated that sex estimation can be made with an accuracy rate exceeding $80 \%$ with equations created by discriminant function analysis from metric measurements made on L1 vertebra [11-12,14-15] (Table 6). However, in our study population, an accuracy rate of more than $80 \%$ could not be obtained in the equations created using metric measurements on the L1 vertebra. Nevertheless, in our study, 
17 measurements that exhibited significant sexual dimorphism were selected for discriminant function analysis, and it was shown that the equation created with six variables could assign sex with an accuracy of $72.6 \%$.

\begin{tabular}{|c|c|c|c|c|}
\hline Author & Populations & Number & Sample & Accuracy (\%) \\
\hline Zheng et al. [11] & Chinese & 210 & CT scan & 88.6 \\
\hline Ostrofsky and Churchill [12] & South African Blacks & 98 & Dry Bone & 87.1 \\
\hline Decker et al. [14] & US North American & 154 & CT scan & 83.8 \\
\hline Azofra-Monge and Alemán Aguilera [15] & Spanish & 94 & Dry Bone & 94.5 \\
\hline Present study & Turkish & 241 & CT scan & 72.6 \\
\hline
\end{tabular}

TABLE 6: Summary of L1 vertebra-based sex estimation studies.

The vertebral body provides strength and support for two-thirds of the vertebral load and is more resistant to mechanical stresses and taphonomic changes with its strong cortical and dense inner trabecular bone structure [18]. Although the possibility of obtaining the entire vertebral column intact is inherently high, the spinous processes and transverse processes of the vertebrae are more affected by taphonomic changes and might be fragmented $[6,18]$. Therefore, it may be more difficult to measure especially on articular facets. In our study population, it was seen that sex estimation could be made with an accuracy of $73.9 \%$ with the equation created from the measurements made only on the vertebral body. It was seen that the accuracy rate of the equation, which was created using the measurements related to the vertebral processes, was $69.7 \%$ in the sex estimation. In previous studies, it is seen that the measurements related to the vertebral body are dominant in the equations created for sex estimation from the L1 vertebra [11-12,15]. In the study of Decker et al. it was stated that the measurements of the vertebral pedicles in the obtained discriminant function equation were more effective in assigning sex [14]. However, in our study, there was a significant decrease in the accuracy rate $(66.8 \%)$ in the equation created using the measurements on the vertebral foramen and pedicles. In our study; the equation created by the measurements made on the L1 vertebral body having a higher accuracy rate compared to other parts of the vertebrae also provides an advantage in terms of the vertebral body being more resistant to taphonomic changes. We think that the data from our study will be useful in cases where L1 vertebrae cannot be obtained as a whole but can be obtained partially. However, in instances where the vertebral column cannot be obtained as a whole or fragmented part of the lumbar vertebrae can be obtained, it may be a problem to determine which vertebra is $\mathrm{L} 1$ or which fragments belong to the L1 vertebra [12].

In our study, it was seen that the equation created with six variables could predict sex with an accuracy of $75.8 \%$ for females, while the accuracy rate was $69.4 \%$ for males. This difference between males and females may be due to the fact that vertebral sizes vary more among males than in females. In our study, it is seen that the standard deviations in the measurements of males are generally larger than the standard deviations of females. A similar situation has also been reported in previous studies examining the lumbar vertebrae [12-13].

The use of different measurement methods in the studies may also lead to differences in the obtained accuracy rates. Different results can be obtained from studies on dry bones and studies using radiological imaging methods. Slice thickness in radiological imaging with CT may also cause differences between studies. When3D reconstruction of anatomical features or other fine details is desired, it is recommended to use a maximum slice thickness of $1.25 \mathrm{~mm}$ [22]. Slice thicknesses greater than $1.25 \mathrm{~mm}$ will cause a loss of fidelity in the 3D reconstruction of images obtained [22]. Although it is recommended to use the narrowest slice thickness possible, it is also stated that there is no significant difference between measurements made using $1.25 \mathrm{~mm}$ slice thickness and measurements made using $0.625 \mathrm{~mm}$ slice thickness [22]. New 3D methods, which are increasingly used, may be useful in demonstrating sexual dimorphism patterns that have not been demonstrated by traditional methods [3]. In addition, radiological methods are also important in terms of developing non-invasive and non-destructive approaches. Although a 3D model is used in the study of Zheng et al. it is stated that measurements are made on-screen images [11]. On the other hand, Decker et al. measured directly from the marker points on the 3D model [14]. In our study, measurements were made directly on the 3D model with a slice thickness of $1 \mathrm{~mm}$. Making the measurements directly on the 3D model will be useful in terms of the obtained study data being a reference for measurements to be made on dry bones.

It has been reported in many studies that skeletal age estimation studies differ between populations $[6,20,23]$. It has also been reported in previous studies that vertebral sizes differ between populations [12,24], as well as an Abstract by Pastor RF (Sexual Dimorphism in Vertebral Dimensions at the 
T12/L1 Junction. Proceedings of the 57th Annual Meeting of the American Academy of Forensic Sciences, Denver, CO; 2005). Therefore, it is a requirement that discriminant function analyses usage in estimating sex from lumbar vertebrae are population specific. Ancestry, genetics, environmental effects, socioeconomic status, secular changes affect the development of the skeletal system. These differences were not taken into account in our study population. Therefore, lower sex prediction rates may have been obtained in our study compared to other studies, or the L1 vertebra in the Turkish population may show less sexual dimorphism compared to other populations. It is necessary to evaluate sexual dimorphism in L1 vertebrae and to establish population-specific discriminant functions by conducting studies in different populations.

\section{Conclusions}

As a result, in our study; with metric measurements made on 3D CT images of the L1 vertebra, it was shown that sex estimation can be made with an accuracy rate of $72.6 \%$ in the Turkish population. Our study is the first study in which L1 vertebrae are examined with the purpose of sex estimation in the Turkish population and we think that our data will be an important reference for sex estimation from L1 vertebra in the Turkish population.

\section{Additional Information \\ Disclosures}

Human subjects: Consent was obtained or waived by all participants in this study. Research Ethics Committee of Tepecik Training and Research Hospital issued approval 2020/11-29. This retrospective study was approved by the Research Ethics Committee of Tepecik Training and Research Hospital (protocol number: 2020/11-29) and the study was conducted in accordance with the standards of the Declaration of Helsinki. Animal subjects: All authors have confirmed that this study did not involve animal subjects or tissue. Conflicts of interest: In compliance with the ICMJE uniform disclosure form, all authors declare the following: Payment/services info: All authors have declared that no financial support was received from any organization for the submitted work. Financial relationships: All authors have declared that they have no financial relationships at present or within the previous three years with any organizations that might have an interest in the submitted work. Other relationships: All authors have declared that there are no other relationships or activities that could appear to have influenced the submitted work.

\section{References}

1. Klepinger L: Fundamentals of forensic anthropology. John Wiley \& Sons, Inc, Hoboken, New Jersey; 2006. $3: 7$.

2. Kemkes-Grottenthaler A: The reliability of forensic osteology - a case in point: case study . Forensic Sci Int. 2001, 117:65-72. 10.1016/s0379-0738(00)00450-3

3. Krishan K, Chatterjee PM, Kanchan T, Kaur S, Baryah N, Singh RK: A review of sex estimation techniques during examination of skeletal remains in forensic anthropology casework. Forensic Sci Int. 2016, 261:165.e1-8. 10.1016/i.forsciint.2016.02.007

4. Forensic Human Identification: An Introduction. Thompson T, Black S (ed): CRC Press, Boca Raton, FL; 2006. 10.1201/9781420005714

5. Franklin D: Forensic age estimation in human skeletal remains: current concepts and future directions . Leg Med (Tokyo). 2010, 12:1-7. 10.1016/j.legalmed.2009.09.001

6. Rozendaal AS, Scott S, Peckmann TR, Meek S: Estimating sex from the seven cervical vertebrae: an analysis of two European skeletal populations. Forensic Sci Int. 2020, 306:110072. 10.1016/j.forsciint.2019.110072

7. Gama I, Navega D, Cunha E: Sex estimation using the second cervical vertebra: a morphometric analysis in a documented Portuguese skeletal sample. Int J Legal Med. 2015, 129:365-72. 10.1007/s00414-014-1083-0

8. Marino EA: Sex estimation using the first cervical vertebra . Am J Phys Anthropol. 1995, 97:127-33. 10.1002/ajpa.1330970205

9. Kibii JM, Pan R, Tobias PV: Morphometric variations of the 7th cervical vertebrae of Zulu, White, and Colored South Africans. Clin Anat. 2010, 23:399-406. 10.1002/ca.20962

10. Wescott DJ: Sex variation in the second cervical vertebra . J Forensic Sci. 2000, 45:462-6. 10.1520/JFS14707J

11. Zheng WX, Cheng FB, Cheng KL, et al.: Sex assessment using measurements of the first lumbar vertebra . Forensic Sci Int. 2012, 219:285.e1-5. https://doi.org/10.1016/j.forsciint.2011.11.022

12. Ostrofsky KR, Churchill SE: Sex determination by discriminant function analysis of lumbar vertebrae . I Forensic Sci. 2015, 60:21-8. 10.1111/1556-4029.12543

13. Oura P, Karppinen J, Niinimäki J, Junno JA: Sex estimation from dimensions of the fourth lumbar vertebra in Northern Finns of 20, 30, and 46 years of age. Forensic Sci Int. 2018, 290:350.e1-6. 10.1016/j.forsciint.2018.07.011

14. Decker SJ, Foley R, Hazelton JM, Ford JM: 3D analysis of computed tomography (CT)-derived lumbar spine models for the estimation of sex. Int J Legal Med. 2019, 133:1497-506. 10.1007/s00414-019-02001-8

15. Azofra-Monge A, Alemán Aguilera I: Morphometric research and sex estimation of lumbar vertebrae in a contemporary Spanish population. Forensic Sci Med Pathol. 2020, 16:216-25. 10.1007/s12024-020-00231-6

16. Standring S: Gray's anatomy: the anatomical basis of clinical practice . Churchill Livingstone, 2008.

17. Hora M, Sládek V: Population specificity of sex estimation from vertebrae. Forensic Sci Int. 2018, 291:279.e1-12. 10.1016/j.forsciint.2018.08.015

18. Waldron T: The relative survival of the human skeleton: implications for palaeopathology . Death, Decay, and Reconstruction: Approaches to Archaeology and Forensic Science. Manchester Univ Pr, Manchester, UK. 1987, 55-64. 


\section{Cureus}

19. Blau S, Briggs CA: The role of forensic anthropology in Disaster Victim Identification (DVI) . Forensic Sci Int. 2011, 205:29-35. 10.1016/j.forsciint.2010.07.038

20. Spradley MK, Jantz RL: Sex estimation in forensic anthropology: skull versus postcranial elements . J Forensic Sci. 2011, 56:289-96. 10.1111/j.1556-4029.2010.01635.x

21. Ulijaszek SJ, Kerr DA: Anthropometric measurement error and the assessment of nutritional status . Br J Nutr. 1999, 82:165-77. 10.1017/s0007114599001348

22. Ford JM, Decker SJ: Computed tomography slice thickness and its effects on three-dimensional reconstruction of anatomical structures. J Forensic Radiol Imaging. 2016, 4:43-6. 10.1016/j.jofri.2015.10.004

23. Gupta SK, Roy RC, Srivastava A: Sagittal diameter of the cervical canal in normal Indian adults . Clin Radiol. 1982, 33:P681-5. 10.1016/s0009-9260(82)80403-0

24. Yu SB, Lee UY, Kwak DS, et al.: Determination of sex for the 12th thoracic vertebra by morphometry of three-dimensional reconstructed vertebral models. J Forensic Sci. 2008, 53:620-5. 10.1111/j.15564029.2008.00701.x 